\title{
Impacts of a cross-institutional undergraduate research experience workshop on student understanding of and self-efficacy for research
}

\author{
Sara Liesman ${ }^{1, *}$, Angela Antonou ${ }^{2}$, Megan Powell $^{3}$ \\ ${ }^{1}$ Department of Mathematics, Illinois State University, Normal, IL, 61761 \\ ${ }^{2}$ Department of Mathematics and Computer Science, University of St. Francis, Joliet, IL, 60435 \\ ${ }^{3}$ Department of Mathematics, University of North Carolina Asheville, Asheville, NC, 28804 \\ sliesma@ilstu.edu
}

There are many perceived benefits to undergraduate student research; however, students may not have a full understanding of the research process prior to engaging in a project. In this paper, we analyze the impact of an undergraduate research workshop on students' understanding of academic research as well as the impact on their self-efficacy for conducting research through an analysis of the 2018 and 2019 Intercollegiate Biomathematics Alliance Cross-Institutional Research Experience (CURE) workshops. Students were asked to complete a survey at the start and end of the three-day workshop, which included both Likert-type scale questions as well as free response questions addressing their understanding of research and effective collaboration in conducting research, their perceived role in conducting research, and their perception of their own skills specific to biomathematics research. We observed improved self-efficacy in specific research skills where students had not already indicated a high level of self-efficacy prior to the workshop. Additionally, student responses indicated a shift in perception of research from solving a particular problem to contributing to a field by discovering or creating new knowledge. Other shifts in student perceptions are discussed in the paper as well as recommendations for the workshop organizers. 